\title{
The hit-and-return system enables efficient time-resolved serial synchrotron crystallography
}

Eike C. Schulz ${ }^{\mathrm{a}}$, Pedram Mehrabi ${ }^{\mathrm{a} \$}$, Henrike M. Müller-Werkmeister ${ }^{\mathrm{a}, \mathrm{b} \$}$, Friedjof Tellkamp ${ }^{\mathrm{c}}$, Ajay Jha ${ }^{a}$, William Stuart ${ }^{\mathrm{a}}$, Elke Persch ${ }^{\mathrm{d}}$, Raoul De Gasparo ${ }^{\mathrm{d}}$, François Diederich ${ }^{\mathrm{d}}$, Emil F. Pai $^{e, f, g, h^{*}}$, R. J. Dwayne Miller ${ }^{a, j, j^{*}}$

aMax-Planck-Institute for Structure and Dynamics of Matter, Department for Atomically Resolved Dynamics, Luruper Chaussee 149, 22761 Hamburg, Germany

bUniversity of Potsdam, Institute of Chemistry, Physical Chemistry, Karl-Liebknecht-Str. 24-25

14476 Potsdam-Golm, Germany

'Max-Planck-Institute for Structure and Dynamics of Matter, Scientific Support Unit Machine Physics, Luruper Chaussee 149, 22761 Hamburg, Germany

'Laboratorium für Organische Chemie, ETH Zürich, Vladimir-Prelog-Weg 3, 8093, Zürich, Switzerland.

eDepartment of Biochemistry, University of Toronto, Toronto, Ontario, Canada

'Department of Medical Biophysics, University of Toronto, Toronto, Ontario, Canada,

${ }^{9}$ Department of Molecular Genetics, University of Toronto, Toronto, Ontario, Canada,

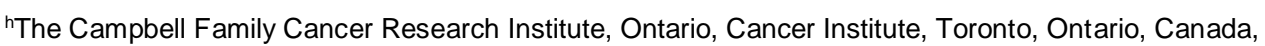

'University of Toronto, Departments of Chemistry and Physics, 80 St. George Street, Toronto, Ontario, M5S 3H6, Canada 'Department of Physics, Centre for Ultrafast Imaging - University of Hamburg, Luruper Chaussee 149, Hamburg, 22761, Germany

\$These authors contributed equally.

"corresponding authors: dwayne.miller@mpsd.mpg.de; emil.pai@utoronto.ca 


\section{Abstract}

We present a method for time-resolved serial synchrotron crystallography with time-resolution from milliseconds to seconds and longer. Timing delays are mechanically set using the regular pattern in fixed-target crystallography chips. Proof-of-principle optical pump-probe experiments demonstrate that data can be collected at synchrotrons and XFELs at short (30 ms), medium ( $752 \mathrm{ms)}$ and long (2052 $\mathrm{ms}$ ) intervals. Therefore, once suitable crystals can be obtained, most enzymes become accessible to time-resolved investigations.

Keywords: time-resolved crystallography, serial synchrotron crystallography (SSX), Pumpprobe experiment, structural enzymology 


\section{Introduction}

Atomically resolved structures of enzymatic reactions acquired at multiple timepoints are required for a full mechanistic understanding of biomolecular catalysis. This is especially true for slower time regimes where most functional dynamic changes take place ${ }^{1}$. Most enzymatic reactions occur on the millisecond to second time-scale and these slow time-scales can be well addressed with the capabilities of current-generation synchrotron sources ${ }^{2}$.

There has been major interest to re-engineer sample delivery methods for serial synchrotron crystallography $(\mathrm{SSX})^{3-6}$. In our fixed target solution, $>20,000$ micro-crystals can be incorporated in lithographic features on a silicon "chip", mounted on high-speed translation stages, enabling a data collection rate of $30 \mathrm{~Hz}$ with up to $120 \mathrm{~Hz}$ possible with current inertia limits ${ }^{7,8}$. Compared to rotation experiments, SSX has the advantage of applying a significantly lower radiation dose to each dataset. This allows for room-temperature experiments at comparably higher resolution, a mandatory prerequisite for time-resolved experiments. Without using pink beam/Laue crystallography, the timeresolution currently achieved at synchrotrons is on the order of milliseconds ${ }^{9,10}$.

Our implementation of this fixed-target serial crystallography solution adds optical excitation for pump-probe experiments. By applying an efficient Hit-And-Return (HARE) system our setup samples short (milliseconds) as well as longer (several seconds to minutes) excitation delays without significantly extending data-collection time. To demonstrate the feasibility of our approach we used fluoroacetate dehalogenase (FAcD) crystals soaked with a photocaged substrate as a model system (Supplementary Fig. 1). FAcD is a remarkably slow enzyme with a turnover rate in the tens of seconds $^{11,12}$. We present structures for four time points $(\mathrm{t}=0 \mathrm{~ms}, \mathrm{t}=30 \mathrm{~ms}, \mathrm{t}=752 \mathrm{~ms}$ and $\mathrm{t}=2,052 \mathrm{~ms}$ ) along the enzyme's reaction coordinate. The simple, robust, general, and highly efficient approach enables TR-SSX on microfocus synchrotron beamlines demonstrating its potential for dynamic biochemical studies. 


\section{Results}

Our modular end-station was reengineered for SSX applied to routine structure determinations ${ }^{8}$

(Supplementary Fig. 2) . Combining it with an optical excitation setup extends its usefulness to TR-

SSX. The novel and versatile HARE approach efficiently exploits the lithographic patterns of crystallography chips. It not only enables millisecond to multi-second TR-SSX, addressing fast and slow time delays with one setup, but also allows for many applications involving pump-probe approaches.

\section{The hit-and-return approach enables time delays from milliseconds to many seconds}

In typical SFX pump-probe experiments in the femtosecond to nanosecond regime, time delays are achieved mechanically by moving an optical delay stage ${ }^{13}$. We introduce a 'HARE approach' based on the regular pattern of the feature positions on crystallography chips in combination with the high speed and accuracy of a translation stage system (motion velocity up to $20 \mathrm{~mm} / \mathrm{s}$ without start/stop). For instance, in HARE 8, (i) the process is started by measuring a dark X-ray image, (ii) directly followed by laser excitation, (iii) performed consecutively at eight positions. (iv) Chip moves back to the first position and (v) for each of the eight positions another X-ray diffraction pattern, this time for the excited state, is recorded. The consecutive delay times are equivalent to the time it takes for the stage to move from position 1 to position 8 and back to position 1 (Fig. 1, Supplementary Fig. 3-7).

The HARE approach can be used for certain integer multiples (Supplementary Fig. 5). In compartments with 24x24 features, time delays corresponding to HARE numbers 2, 3, 4, 6, 8, 12, 24, 48, 72, 96, 144, 288 and longer (e.g. HARE 576) are feasible (Fig. 2). This method represents a simple and flexible approach for TRX covering large time windows. A brief description of suitable chip geometries, valid HARE constants, and formulae to generate chip coordinates for a given HARE constant are given in Supplemental Fig. 5. Data for a time delay faster than HARE 2 are acquired with standard (i.e. non HARE) pump-probe delay after excitation.

\section{Proof-of-Principle}

To demonstrate the feasibility of our setup for TR-SSX for varying time delays, we have used 
Rhodopseudomonas palustris fluoroacetate dehalogenase (FAcD) as a model system

(Supplementary Fig. 1). The homodimeric FAcD catalyses the hydrolysis of the pesticide fluoroacetate albeit with a very low turnover number ${ }^{12}$. The $\mathrm{S}_{\mathrm{N}} 2$ hydrolysis of the C-F bond involves binding of the substrate to the apo-protein, formation of a covalent intermediate, followed by hydrolysis of the intermediate and product release as the final step. To establish a process suitable to probe FAcD's catalytic substeps, microcrystals were soaked with photocaged fluoroacetate (pHPFAc) $)^{14}$, which upon exposure to $320-360 \mathrm{~nm}$ light undergoes fast photo-cleavage to the substrate fluoroacetate. Structures at four time points along the reaction coordinate pathway, namely dark state, $30 \mathrm{~ms}$ state, $752 \mathrm{~ms}$ and $2052 \mathrm{~ms}$ after exposure, clearly show that the results are reproducible $\left(\mathrm{t}_{0}\right.$ and $\mathrm{t}_{30 \mathrm{~ms}}$ ), that electron density and flexibility changes occur as a function of time, and that they can be observed ( $\mathrm{t}_{752 \mathrm{~ms}}$ and $\mathrm{t}_{2052 \mathrm{~ms}}$ ) (Fig. 2, Supplementary Figs. 8, 9).

Consistent with previously identified half-the-sites reactivity ${ }^{12}$, the $752 \mathrm{~ms}$ structure clearly displays the ligand (fluoroacetate) in the active site of subunit A. This unambiguously shows that the substrate can be released from the caged compound and specifically binds to the active site of FAcD. At $2052 \mathrm{~ms}$, changes in the electron density of ligand and Tyr149 can be interpreted as structural rearrangements in line with substrate turnover (Fig. 2, Supplementary Figs. 8, 9). Comparison of the electron density maps at $0 \mathrm{~ms}$ and $30 \mathrm{~ms}$ shows the good reproducibility of the results. Differences between dark state and $752 \mathrm{~ms}$ and $2052 \mathrm{~ms}$ states reveal additional density in the active site as expected for substrate binding - and potential intermediate formation (Fig. 2, Supplementary Figs. 8, 9). Increasing substrate dynamics at the $2052 \mathrm{~ms}$ time point suggests that the ligand samples multiple states before achieving the correct geometry for an $\mathrm{S}_{\mathrm{N}} 2$ attack. It seems that different time points leading up to the formation of the covalent intermediate will encompass different positional states of the substrate. Compared to the static mutant structures, which represent fully formed low energy states, these time-points provide insight into real-time dynamics, difficult to observe with other crystallographic methods ${ }^{12}$. Likewise, a significant reduction of electron density can be observed for the cap domain of chain A, aligning with a previously determined increase in flexibility of this domain during substrate turnover. POLDER omit, END-RAPID and $\mathrm{F}_{\mathrm{o}}-\mathrm{F}_{\mathrm{o}}$ maps for these regions show the same changes in electron density (Fig. 2, Supplementary Figs. 8, 9) ${ }^{12}$. Arguments that these results are not caused by radiation damage are 
presented in Supplementary Results and Discussion (Supplementary Figs. 10,11).

\section{Discussion}

We describe a versatile setup to conduct TR-SSX diffraction experiments on a wide range of timescales. Limitations with respect to fraction excited and signal size are comparable to other lightinduced TRX experiments ${ }^{15-17}$. The method depends on a stable, high-flux microfocus synchrotron beamline with a high frequency counting detector to ensure sufficient brightness and temporal resolution. Photochemistry for reaction initiation also needs to be sufficiently fast. The system is restricted to sampling discrete time-points, as the time-structure is mechanically encoded in the chip pattern and the acceleration of the translation stages. However, various HARE time delays (e.g. 2 288), together with a simple software switch, allow capturing data in a wide temporal range (Fig. 2B), covering the complete turnover cycle of most, including some of the slowest enzymes. Once interesting structures are captured they can be further analysed via fine time-slicing between the discrete HARE time points by adding a software delay between the features of the chip, thereby enabling data collection on a full gamut of time points above a beamline-specific minimum to study complex kinetic behaviour. Although the system's design is optimized for synchrotrons, it is easily transferrable to XFELs if faster time points are targeted. While limitations are similar to those of other methods in the field, its clear advantage lies in its simplicity, which adds to robustness and reliability. It can obtain many time-points efficiently for data acquisitions at rates $<120 \mathrm{~Hz}^{8}$. Moreover, a TTLpulse is sufficient for signal synchronisation.

Since the average rate of the majority of enzymatic reactions is $\sim 10^{-1} \mathrm{~s}$, the time-window our setup offers is well posed to record stop-gap movies of biology in action ${ }^{1}$. The current liquid jet technology cannot easily provide access to these slow time-points without hardware modifications ${ }^{18,19}$. While conveyor-belt approaches can address longer time-delays the problem to reduce extensive datacollection times has not been addressed, yet ${ }^{20}$. Relative to other current technologies, the matrix scheme of our fixed-target solution offers the greatest temporal flexibility in probing a multitude of time points, from short to very long. Without the HARE approach, the $2052 \mathrm{~ms}$ time point would require > 12 hours of data collection per chip; applying the HARE approach reduces this to $\sim 1$ hour. 
This displays the main advantage of the described TR-SSX approach, which allows one to collect enough time-points within a single beam-time to reconstitute a "stop-gap molecular movie" that samples a broad time scale and thereby encompasses the reaction coordinate pathway of potentially many enzymes.

In conclusion, we present a widely applicable and highly versatile setup for TR-SSX that can sample the predominant time-scales of enzymatic reactions. It is applicable especially to all light-triggered systems but could also be exploited for $\mathrm{pH}$ or T-jump reaction initiations. Fully exploiting this system should eventually provide us with the opportunity to record a molecular movie of a full enzymatic catalysis cycle on a biologically relevant time-scale. 


\section{Acknowledgements}

Synchrotron MX data were collected at beamline P14 operated by EMBL Hamburg at the PETRA III storage ring (DESY, Hamburg, Germany). The authors thank Thomas Schneider and Gleb Bourenkov for exceptional beamline support and together with Arwen Pearson for discussions of the experiment. The Authors are grateful to Wolfgang Kabsch and Kay Diederichs for outstanding support in data processing and helpful discussions. Parts of this research were also carried out at beamline P11 at PETRA III at DESY, a member of the Helmholtz Association (HGF). We would like to gratefully recognize Anja Burkhardt and Olga Lorbeer for assistance in using P11. We thank Ladislav Andricek of the Max-Planck Semiconductor lab for fabrication of the crystallography chips. The authors gratefully acknowledge the support provided by the Max Planck Society and the excellence cluster "The Hamburg Centre for Ultrafast Imaging - Structure, Dynamics and Control of Matter at the Atomic Scale" of the Deutsche Forschungsgemeinschaft (R.J.D.M.) and the People Programme (Marie Curie Actions) of the European Union's Seventh Framework Programme (FP7/2007-2013) under REA grant agreement no. 623994 (H.M.-W.). P.M. is the recipient of an Alexander von Humboldt-Stiftung postdoctoral fellowship. Work at the ETH was generously supported by the ETH Research Council (ETH-01 13-2) (F.D.). E.F.P. gratefully acknowledges funding from the Natural Sciences and Engineering Research Council of Canada (award No. RGPIN-2015- 04877) and the Canada Research Chairs program as well as the Burroughs Wellcome Fund for a Collaborative Research Travel Grant.

\section{Author Contributions}

E.C.S., P.M., H.M.-W., E.F.P. and R.J.D.M. designed the experiment. E.C.S., P.M. and H.M.-W. performed the experiments with support from F.T., E.F.P and W.S.; E.P, R.DG. and F.D. designed a reaction scheme and synthesized the caged compound. P.M. prepared the protein crystals. F.T. designed the experimental endstation and together with P.M. developed the HARE algorithms. H.M.-W. and A.J. built the optics part of the setup. E.C.S. and P.M. analysed and processed the diffraction data and wrote the manuscript. All authors discussed and corrected the manuscript.

\section{Competing Financial Interests Statement}

The authors declare no competing financial interests. 


\section{References}

1. Bar-Even, A. et al. The moderately efficient enzyme: evolutionary and physicochemical trends shaping enzyme parameters. Biochemistry 50, 4402-4410 (2011).

2. Fenwick, R.B., Esteban-Martin, S. \& Salvatella, X. Understanding biomolecular motion, recognition, and allostery by use of conformational ensembles. Eur Biophys J Biophy $\mathbf{4 0}$, 1339-1355 (2011).

3. Gati, C. et al. Serial crystallography on in vivo grown microcrystals using synchrotron radiation. Iucrj 1, 87-94 (2014).

4. Standfuss, J. \& Spence, J. Serial crystallography at synchrotrons and X-ray lasers. Iucrj 4 , 100-101 (2017).

5. Stellato, F. et al. Room-temperature macromolecular serial crystallography using synchrotron radiation. IUCrJ 1, 204-212 (2014).

6. Weinert, T. et al. Serial millisecond crystallography for routine room-temperature structure determination at synchrotrons. Nature Communications 8 (2017).

7. Oghbaey, S. et al. Fixed target combined with spectral mapping: approaching $100 \%$ hit rates for serial crystallography. Acta Crystallogr D Struct Biol 72, 944-955 (2016).

8. Sherrell, D.A. et al. A modular and compact portable mini-endstation for high-precision, highspeed fixed target serial crystallography at FEL and synchrotron sources. Journal of Synchrotron Radiation 22, 1372-1378 (2015).

9. Anfinrud, P.A., Lim, M. \& Jackson, T.A. Structure, dynamics, and function of proteins: New insights from time-resolved IR spectroscopy. Prog Biophys Mol Bio 65, Sa302-Sa302 (1996).

10. Schotte, F. et al. Watching a protein as it functions with 150-ps time-resolved X-ray crystallography. Science 300, 1944-1947 (2003).

11. Chan, P.W., Yakunin, A.F., Edwards, E.A. \& Pai, E.F. Mapping the reaction coordinates of enzymatic defluorination. J Am Chem Soc 133, 7461-7468 (2011).

12. Kim, T.H. et al. The role of dimer asymmetry and protomer dynamics in enzyme catalysis. Science 355 (2017).

13. Kubo, M. et al. Nanosecond pump-probe device for time-resolved serial femtosecond crystallography developed at SACLA. Journal of Synchrotron Radiation 24, 1086-1091 (2017).

14. Givens, R.S. et al. p-Hydroxyphenacyl photoremovable protecting groups - Robust photochemistry despite substituent diversity. Can J Chem 89, 364-384 (2011).

15. Levantino, M., Yorke, B.A., Monteiro, D.C.F., Cammarata, M. \& Pearson, A.R. Using synchrotrons and XFELs for time-resolved X-ray crystallography and solution scattering experiments on biomolecules. Curr Opin Struc Biol 35, 41-48 (2015).

16. Spence, J.C.H. Approaches to time-resolved diffraction using an XFEL. Faraday Discuss 171, 429-438 (2014).

17. Spence, J.C.H. XFELs for structure and dynamics in biology. IUCrJ 4, 322-339 (2017).

18. Chavas, L.M., Gumprecht, L. \& Chapman, H.N. Possibilities for serial femtosecond crystallography sample delivery at future light sources. Struct Dyn 2, 041709 (2015).

19. Olmos, J.L., Jr. et al. Enzyme intermediates captured "on the fly" by mix-and-inject serial crystallography. BMC Biol 16, 59 (2018).

20. Beyerlein, K.R. et al. Mix-and-diffuse serial synchrotron crystallography. IUCrJ 4, 769-777 (2017). 


\section{Figure legends}

\section{Figure 1 - The HARE approach.}

A representation of a single compartment on the chip with $24 \times 24$ features. X-ray pulses are depicted in green, while laser pulses are shown in blue. In order to have a large number of valid HARE times, it is most efficient that the number of features per compartment row and column is a highly composite number (e.g. 24). Larger time-points than a HARE value of 24 would therefore need to subsequently be certain multiples of 24 (ie. $24,48,72 \ldots$ ).

Data collection for a HARE of 8 would be as follows i) Starting from row 'a', the first feature (aa) is exposed to an X-ray pulse and a subsequent dark image is recorded. Immediately following the $\mathrm{X}$-ray pulse the sample is pumped with the fs-laser for $\mathrm{t}=0$. This procedure is repeated for a total of 8 features. ii) Upon pumping the 8 features the stages move back to their starting positions. iii) The same 8 features are then re-probed with X-rays after a delay time matching the total time for probe-pumping 8 features. For every probed feature on the chip, the resulting data include both a dark image and an exposed image with a corresponding delay dependent on the HARE number. In Hit-and-Return the data collection time is optimized by reference data collection $\left(\mathrm{t}_{0}\right)$ and reaction initiation (pump) of further crystals during the delay time. After the delay time the system returns to the initial position to collect the probe data. The delay time is defined by variable parameters the pitch of the features, the translation time, the X-ray exposure and the Laser exposure all of which are summarized in a single Hit-and-Return number. The longer the delay time should be the more features can be pumped before a probe run the higher the Hit-and-Return number. By contrast, using sequential delays the delay times would accumulate to much longer data-collection times. This effect is more pronounced for longer delay times. Please refer to Supplemental Results - Comparison of sequential and HARE time delays.

\section{Figure 2 - Time delays of the FAcD catalytic cycle trapped by the HARE approach.}

a) Pictorial representation of the FAcD catalytic cycle. The various stages of FAcD's catalytic cycle are represented with various ligands and intermediates highlighted in the active site. The 
blue region of cycle represents the time range covered by our TR-SSX. It captures the Michaeliscomplex (752 ms) and increased substrate and active site dynamics (2052 ms) using the HARE approach. The red region consists of anticipated states along the reaction coordinate pathway, namely: the covalent intermediate (PDB-ID: 5K3F) and the product complex (PDB-ID: 5K3E), which were captured via mutant trapping. These two distinct stages, together with more finely sliced time points, along the catalytic cycle are yet to be measured using the HARE approach.

b) HARE schematic for time delays. Pictorial representation of the time-delays for the HARE approach from 2 to 288. (*) represents a HARE time delay for arbitrarily long time points (e.g. 576). The corresponding table represents the various HARE time delays and their matching time points for a $12 \times 12$ and $24 \times 24$ chip design.

c) FAcD electron density changes as a function of time - all electron density maps are represented as POLDER omit maps at $2 \sigma$ cutoff (green - protein, blue - ligand). (0 MS) Before the photo caged substrate is released by the fs-laser pulse the active site is mostly empty only showing minor blobs of electron density likely corresponding to water molecules. Both cap-domains show equivalent electron density distribution. (30MS) - Only minor changes can be identified in the electron density map in the active site (probably a water molecule moving slightly). First reduction of electron density can be observed for the capdomain of subunit B. (752MS) - The active is occupied with a ligand molecule as represented by clearly defined electron density. The electron density for the cap-domain in subunit B is further reduced. (2052MS) - A clear change in the active site electron density corresponding to the ligand is visible $2052 \mathrm{~ms}$ after the excitation, presumably reflecting an increase in active site dynamics as displayed by the altered electron density morphology. We hypothesise that this ligand orientation approaches covalent intermediate formation in the active site. While the electron density in the cap domain of chain A is almost identical in maps calculated from data collected at $\mathrm{t}=0 \mathrm{~ms}$ and $\mathrm{t}=30 \mathrm{~ms}$ it is significantly reduced after $\mathrm{t}=752 \mathrm{~ms}$ and $\mathrm{t}=2,052$ ms indicating strong mobility of this part of the polypeptide chain. This is consistent with previous reports describing an increased flexibility of this region during substrate turnover 12. 


\section{Online Methods}

\section{Protein crystallization and sample preparation}

Recombinant fluoroacetate dehalogenase (FAcD) was purified from Escherichia coli BL21(DE3) as described $^{11,12}$. FAcD was extracted from $E$. coli cell-free lysate using Ni-chromatography with subsequent cleavage of the His ${ }_{6}$-tag using TEV protease. Size exclusion chromatography was performed using $50 \mathrm{mM}$ Tris- $\mathrm{H}_{2} \mathrm{SO}_{4}$ and $150 \mathrm{mM} \mathrm{NaCl}$, followed by buffer exchange to remove $\mathrm{NaCl}$ as a final step in purification. FAcD crystals were grown in crystallization buffer $(18-20 \%(\mathrm{w} / \mathrm{v}))$ PEG3350, $200 \mathrm{mM} \mathrm{CaCl}_{2}$, and $100 \mathrm{mM}$ Tris- $\mathrm{HCl} \mathrm{pH}$ 8.5). These crystals were used to generate a microseed stock using a 4-8\% higher PEG3350 concentration and a seed bead kit from Hampton Research (HR2-320). Microcrystals were produced using batch crystallization; 100-200 $\mu$ l of both seed stock and $0.5 \mathrm{mM}$ FAcD solution were mixed in a 1:1 ratio. Crystals grew to approximately 2$150 \mu \mathrm{m}$ in size in one to three days.

\section{Synthesis of caged fluoroacetate}

The synthesis and full characterization of the previously unknown photocaged fluoroacetate are reported in the Supplementary Notes.

\section{Chip loading}

A suspension of 100-200 $\mu 1$ crystals of FAcD with an average crystal size of ca. $20 \mu \mathrm{m}$ was equilibrated with pHP-FAc in a crystal stabilizing solution. It was then loaded onto a single chip by applying moderate vacuum suction as described previously ${ }^{7,21-23}$.

\section{Experimental setup and data collection}

For serial data collection, a portable, modular end-station was mounted to the diffractometer similar to the one described in $7,8,21-26$. The end-station is comprised of high-speed precision translation stages (SmarAct) and a home-built controller unit. For the time-resolved experiments described here, a femtosecond laser source (Pharos SP-1.5-200kHz, fundamental = $1030 \mathrm{~nm}$ ) with an integrated pulse 
picker was used for excitation. A home-built portable optical setup for second harmonic generation (SHG, $515 \mathrm{~nm}$ ) and third harmonic generation (THG, $343.3 \mathrm{~nm}$ ) and a non-linear optical parametric amplifier (NOPA) allowed flexible pump wavelengths. For the described experiments the THG was used as pump light. The beam was guided via a periscope onto a small breadboard next to the endstation (Supplementary Fig. 2) and focused onto the sample. The X-ray beam and the laser pump beam where arranged in non-collinear geometry with an angle of approximately $25^{\circ}$. The X-ray focus was approximately $5 \times 9 \mu \mathrm{m}$ and the laser focus approximately $90 \times 90 \mu \mathrm{m}$ (FWHM) with an energy of $45 \mathrm{~nJ} /$ pulse. Spatial overlap of both beams was achieved by alignment onto a thin Ce:YAG crystal that was positioned in the same plane as the crystallography chip. The laser excitation was set to $45 \mathrm{~nJ}$ for a spot size of $100 \mu \mathrm{m}$ (FWHM). The beam was subsequently sent through the crystallography chip, with the bottom aperture acting as effective aperture for the laser excitation to a size of $8 \mathrm{x} 8 \mu \mathrm{m}$. The effectively excited crystal volume was approximately $8 \times 8 \mu \mathrm{m}$ x crystal thickness of $20 \mu \mathrm{m}$. Taking into account variations in crystal thickness and absorptivity at the excitation wavelength, we estimate the mean fraction excited achieved is between $10-15 \%$.

In addition to the previously described setup, a near-infrared imaging system was developed (IDS UI1240SE camera without IR cut-off filter and a self-made $35 \mathrm{~mm}$ objective lens) to allow for chip alignment without visible light. This allows the study of photosensitive crystal systems without disturbance by alignment light.

Diffraction data were collected at room temperature (294 K) at PETRA III at EMBL beamline P14 and beamline P11 at DESY, Hamburg. Crystals were not rotated during an exposure and still images were recorded at a wavelength of $0.976 \AA ̊$ using a Pilatus3 6M detector. A crystal to detector distance of $270 \mathrm{~mm}$ was used during data collection. The detector exposure time was set to $15 \mathrm{~ms}$. Instead of shuttering the X-ray beam, we moved the chip in and out of position to avoid overexposure of the protein crystals in the X-ray beam. Crystals were exposed >= $15 \mathrm{~ms}+2 \mathrm{~ms}$ for the dark image and again $15 \mathrm{~ms}+2 \mathrm{~ms}$ for the excited image (Supplementary Fig. 4). After exposure and the triggered detection time had passed, the chip was moved such that only the silicone material was hit by the Xrays during data collection. We have addressed further details such as extension of the mini end station with an IR viewing system, the optical excitation and indirect shuttering, the comparison of sequential 
and HARE time delays as well as the accuracy of the timing in the Supplementary Results and Discussion.

\section{Data processing}

Diffraction data were processed using the nXDS package (Supplementary Table 1, 2) ${ }^{27}$. The structure was solved by molecular replacement in PHASER ${ }^{28}$ using a previously determined FAcD structure as a search model (PDB-ID: 3R3U). Structure refinement was completed by iterative cycles of refinement in phenix.refine ${ }^{29}$ and manual model building of additional and disordered residues in $\mathrm{COOT}^{30}$. Groups for TLS refinement were generated using the TLSMD webserver ${ }^{31}$. POLDER-OMIT maps were generated using the PHENIX package ${ }^{32}$. Absolute value maps were generated using the END-RAPID software ${ }^{33}$. Molecular images were generated in PyMOL ${ }^{34}$.

\section{Data availability and Accession Code availability}

The software and the GUI to control the hardware and set the HARE numbers is available at https://github.com/pmehrabi/HARE. RCSB accession codes are listed in Supplementary Table 1. 


\section{Methods only References}

21. Owen, R.L. et al. Low-dose fixed-target serial synchrotron crystallography. Acta Crystallographica Section D-Structural Biology 73, 373-378 (2017).

22. Schulz, E.C. et al. Protein crystals IR laser ablated from aqueous solution at high speed retain their diffractive properties: applications in high-speed serial crystallography. Journal of Applied Crystallography 50, 1773-1781 (2017).

23. Zarrine-Afsar, A. et al. Crystallography on a chip. Acta Crystallogr D Biol Crystallogr 68, 321-323 (2012).

24. Epp, S.W. et al. Time zero determination for FEL pump-probe studies based on ultrafast melting of bismuth. Struct Dynam-Us 4 (2017).

25. Mueller, C. et al. Fixed target matrix for femtosecond time-resolved and in situ serial microcrystallography. Struct Dynam-Us 2 (2015).

26. Muller-Werkmeister, H.M. et al. Capturing Functionally Relevant Protein Motions at the Atomic Level: Femtosecond Time Resolved Serial Crystallography of Ligand Dissociation of Carboxy-Myoglobin. Biophys J 110, 513a-513a (2016).

27. Kabsch, W. Processing of X-ray snapshots from crystals in random orientations. Acta Crystallogr D Biol Crystallogr 70, 2204-2216 (2014).

28. McCoy, A.J. et al. Phaser crystallographic software. J Appl Crystallogr 40, 658-674 (2007).

29. Adams, P.D. et al. PHENIX: a comprehensive Python-based system for macromolecular structure solution. Acta Crystallogr D Biol Crystallogr 66, 213-221 (2010).

30. Emsley, P., Lohkamp, B., Scott, W.G. \& Cowtan, K. Features and development of Coot. Acta Crystallogr D 66, 486-501 (2010).

31. Painter, J. \& Merritt, E.A. Optimal description of a protein structure in terms of multiple groups undergoing TLS motion. Acta Crystallogr D Biol Crystallogr 62, 439-450 (2006).

32. Liebschner, D. et al. Polder maps: improving OMIT maps by excluding bulk solvent. Acta Crystallogr D Struct Biol 73, 148-157 (2017).

33. Lang, P.T., Holton, J.M., Fraser, J.S. \& Alber, T. Protein structural ensembles are revealed by redefining X-ray electron density noise. Proc Natl Acad Sci U S A 111, 237-242 (2014).

34. Schrodinger, LLC (2015). 